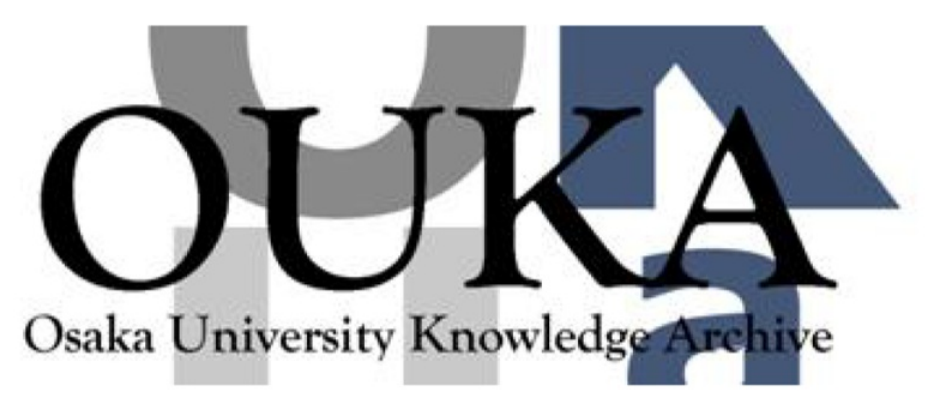

\begin{tabular}{|c|l|}
\hline Title & $\begin{array}{l}\text { A Wide Dynamic Range Variable Gain Amplifier } \\
\text { with Enhanced IP1 dB and Temperature } \\
\text { Compensation }\end{array}$ \\
\hline Author(s) & $\begin{array}{l}\text { Sato, Hisayasu; Maruyama, Takaya; Matsuoka, } \\
\text { Toshimasa et al. }\end{array}$ \\
\hline Citation & $\begin{array}{l}\text { IEICE Transact ions on Electronics. E94-C(8) } \\
\text { p. 1311-p. 1319 }\end{array}$ \\
\hline Issue Date & $2011-08$ \\
\hline oaire:version & VoR \\
\hline URL & https://hdl. handle. net/11094/51681 \\
\hline rights & copyright $\odot 2011$ IEICE \\
\hline Note & \\
\hline
\end{tabular}

Osaka University Knowledge Archive : OUKA

https://ir. Library. osaka-u. ac. jp/

Osaka University 


\title{
A Wide Dynamic Range Variable Gain Amplifier with Enhanced IP1 dB and Temperature Compensation
}

\author{
Hisayasu SATO $^{\dagger, \dagger \dagger a)}$, Takaya MARUYAMA ${ }^{\dagger}$, Toshimasa MATSUOKA $^{\dagger \dagger}$, and Kenji TANIGUCHI $^{\dagger \dagger}$, Members $^{\circ}$
}

\begin{abstract}
SUMMARY This paper presents the design consideration of a fourstage variable gain amplifier (VGA) with a wide dynamic range for receivers. The VGA uses parallel amplifiers for the first and second amplifiers in order to improve the input third-order intercept point (IIP3) in the low gain region. To investigate the behavior of the VGA, the gain and linearity analyses are newly derived for the parallel amplifiers, and are compared with the measured results. In addition, the principle of the temperature compensation is described. The gain control range of $110 \mathrm{~dB}$, the IP1 dB of $-11 \mathrm{dBm}$, and noise figure $(\mathrm{NF})$ of $5.1 \mathrm{~dB}$ were measured using a $0.5 \mu \mathrm{m}$ $26 \mathrm{GHz} \mathrm{f}_{\mathrm{T}}$ BiCMOS process.

key words: VGA, $1 \mathrm{~dB}$ compression point, noise figure, wireless, transceiver, IIP3
\end{abstract}

\section{Introduction}

Variable gain amplifiers (VGAs) are indispensable building blocks for wireless transceivers in order to maximize the dynamic range of the overall systems. The VGAs for receivers are typically used in an automatic gain control loop in wireless systems, and adjust the received signal amplitude so as not to overflow the input dynamic range of $A / D$ converters. For many wireless applications, such as Wideband Code Division Multiple Access (WCDMA), the receivers have to handle unpredictable receiver power which has an 80 to $100 \mathrm{~dB}$ range [1]-[3]. Therefore, the VGAs have to satisfy both high linearity at low gain and low noise at high gain. In addition, to achieve precise gain control and avoid signal phase discontinuity during gain settling, ana$\log$ VGAs with monotonic gain control characteristics are preferred.

The basic concept of an analog VGA with linear-in-dB gain characteristics is introduced in Ref. [4]. A vast amount of the VGAs reported so far are based on this circuit topology. Figure 1 shows a conventional variable gain amplifier (Type 1) using a bipolar quad. The output current of the lower differential pair is controlled by current steering with the upper differential pair. The current transfer of the upper differential pair can be written as

$$
\frac{I_{c 1}}{I_{e}}=\frac{1}{1+\exp \left(-V_{c n t} / V_{T}\right)}
$$

Manuscript received December 8, 2010.

Manuscript revised April 1, 2011.

$\dagger$ The authors are with Renesas Electronics Corp., Itami-shi, 664-0005 Japan.

${ }^{\dagger \dagger}$ The authors are with the Division of electrical, Electronics and Information Engineering, Osaka University, Suita-shi, 5650871 Japan.

a) E-mail: hisayasu.sato.jc@ renesas.com

DOI: 10.1587/transele.E94.C.1311

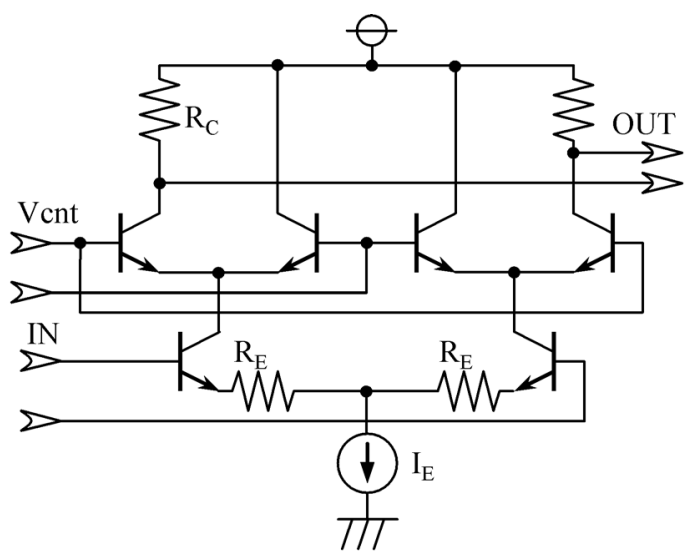

Fig. 1 Conventional VGA (type 1).

$$
\cong \exp \left(V_{c n t} / V_{T}\right)\left(-V_{c n t} / V_{T} \gg 1\right),
$$

where $V_{T}(=\mathrm{kT} / \mathrm{q})$ is the thermal voltage, and $V_{c n t}, I_{c 1}$ and $I_{e}$ are the control voltage to the upper differential pair, one side of the collector current of the upper differential pair, and the emitter current or the output current of the lower differential pair, respectively. As shown in Eq. (1), it is easy to make linear-in-dB characteristics using the bipolar quad, and it can cover 20 to $30 \mathrm{~dB}$ of gain variation. That is why this circuit topology is widely used.

On the other hand, the conventional VGA can not avoid the substantial problem of noise and linearity tradeoff because input linearity, such as the input third-order intercept point (IIP3), and NF, are mainly defined and fixed by the emitter degeneration resistor $R_{E}$. Both a small NF for a small input signal and a high IIP3 for a large input signal are required for wireless applications.

One solution for this problem has been proposed in Ref. [3]. Since it did not show the design consideration for linearity, we will discuss an analysis of the gain and linearity of the VGA in detail in this paper. In addition, temperature compensation will be described.

In the following section, we introduce the wide dynamic range VGA with parallel amplifiers proposed in Ref. [3] again. Then the voltage gain and linearity of the VGA are discussed using simple circuit analysis. The principle of the temperature compensation is also described. In Sect. 3, the measurement results of the VGA and a comparison between the calculated and simulated results are presented. The conclusion is given in the final section. 


\section{VGA Design}

\subsection{VGA Circuit}

One effective solution to overcome the noise and linearity tradeoff problem mentioned in the introduction is to use two amplifiers, which have different characteristics to each other, in parallel. Figure 2 shows a simplified circuit of the parallel-amplifier VGA (Type 2). The VGA has two amplifiers, a high-gain amplifier (HGA) and a low-gain high-linear amplifier (HLA). The differential pair without an emitter degeneration resistor is the HGA, while the other pair is the HLA. The output currents of the HGA and the HLA are combined at load resistors $R_{C}$. The control voltage $V_{c n t}$ for these amplifiers changes complementally. The gain of the HGA grows as the $V_{c n t}$ rises, while the gain of

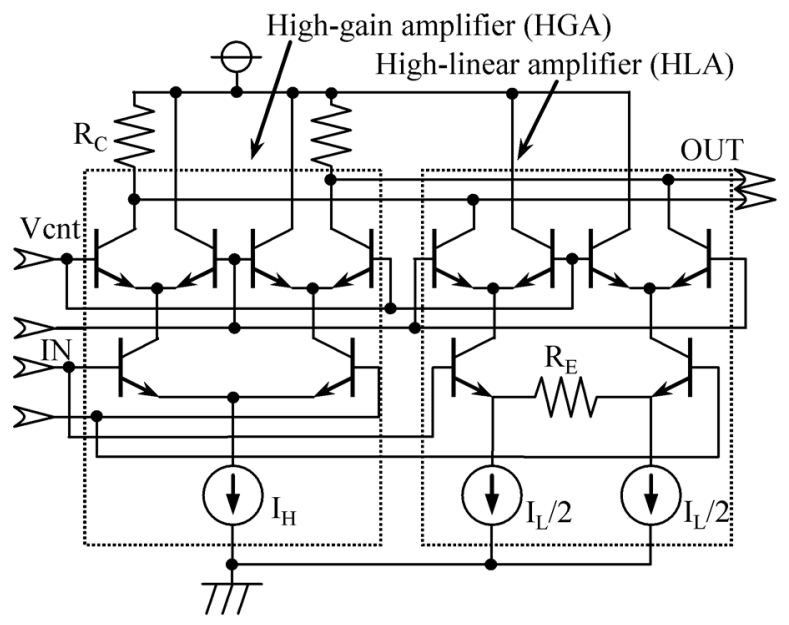

Fig. 2 Parallel-amplifier VGA (type 2). the HLA becomes smaller. Although this circuit topology aims at a high IIP3 at low gain and low noise at high gain, the VGA has another problem. Since the HGA has a much larger gain than the HLA, the third-order distortion current generated by the HGA becomes dominant. Therefore, the total IIP3 of the VGA decreases suddenly when the total gain exceeds the minimum gain by even a little.

Figure 3 shows the VGA proposed in Ref. [3] for the first and second stages. The HGA and HLA of the first stage (HGA1 and HLA1) are fully separated and paralleled, and have a common input and different outputs. Since HGA1 and HLA1 have their own load resistors $\left(R_{C 1 H}\right.$ and $\left.R_{C 1 L}\right)$, the gain and linearity of each amplifier can be designed and optimized independently. The gain control is done by the variable current sources $I_{1 H}$ and $I_{1 L}$ instead of the upper differential pair. The $I_{1 H}$ and $I_{1 L}$ are complementary, and generated by a differential amplifier, which will be described in Sect. 2.4. This configuration has the advantages of large voltage headroom, or high linearity, and small current consumption at low gain. The tail current of HGA1 is large for low noise, and is reduced at low gain. The second stage HGA and HLA (HGA2 and HLA2) follow HGA1 and HLA1, respectively. The second stage is a Type 2 VGA. The output currents of the HGA2 and the HLA2 are summed at the output load resistors $R_{C 2}$.

Figure 4 shows a block diagram of the 4-stage VGA, where the first and second stages are Type 3 VGA. Conventional Type 1 VGAs are used for the third and fourth stages because the noise and linearity requirements are more relaxed at the input of the third stage. Note that the control voltage $V_{c n t}$ for the high-gain path and the low-gain path is complementary to obtain high linearity at low gain. Each stage of the four stages is connected by capacitor coupling to avoid saturation of the amplifiers due to DC offset.

The advantages of this configuration are as follows.

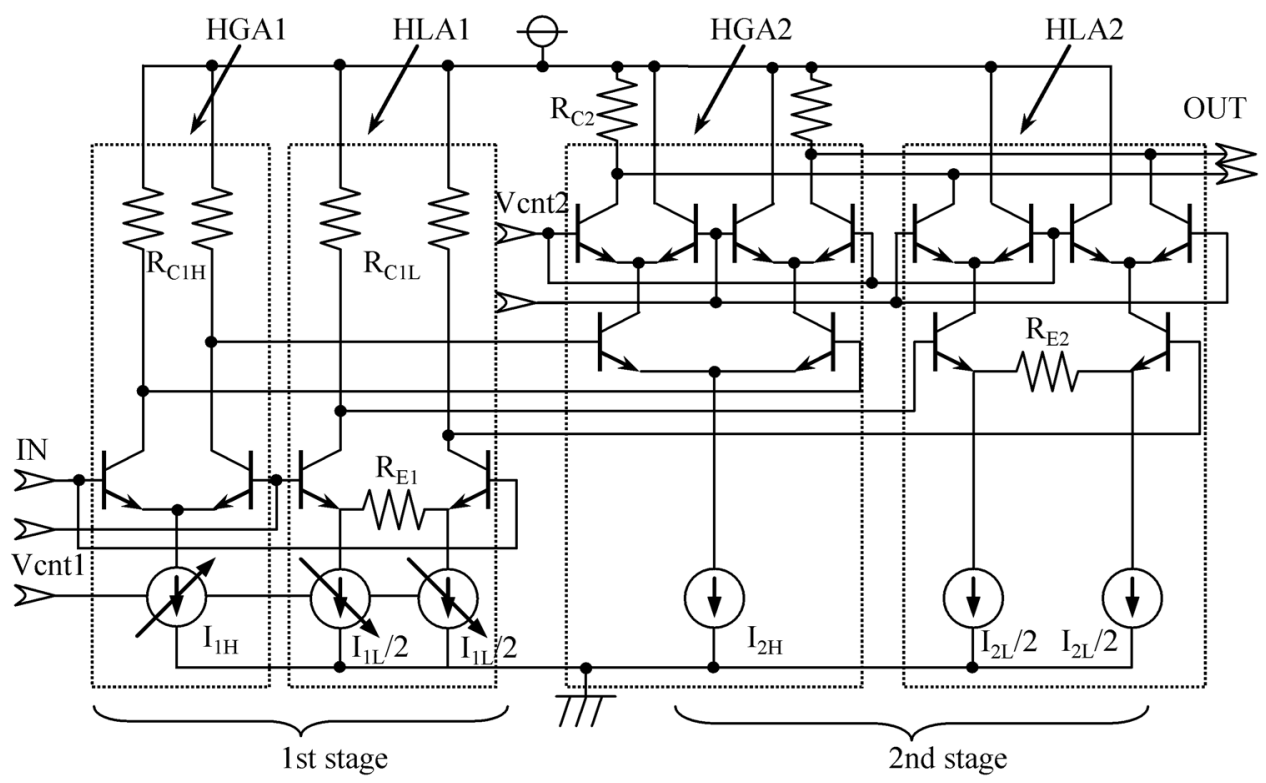

Fig. 3 Fully parallel-amplifier VGA (type 3). 


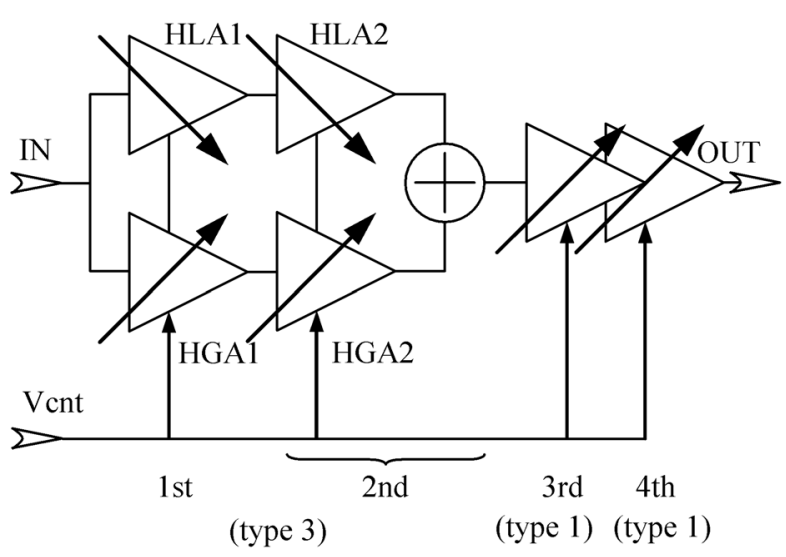

Fig. 4 Block diagram of the 4-stage VGA.

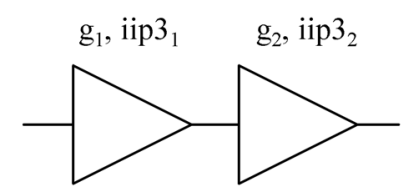

(a)

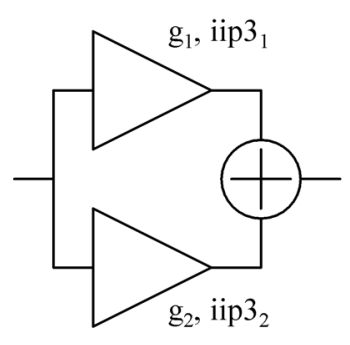

(b)

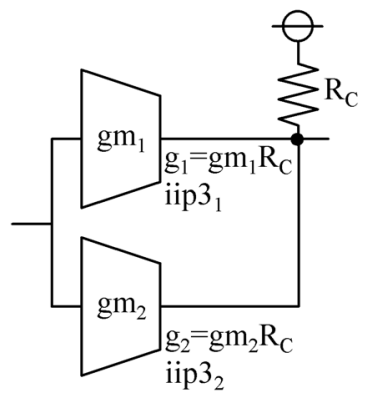

(c)
Fig. 5 (a) Cascaded amplifter, (b) parallel amplifter, (c) simplified circuit of the parallel amplifter.

First, the noise, linearity, and current consumption of the first stage can be optimized. Second, the distortion current of the HGA1 at low gain and the noise current of the HLA1 at high gain are suppressed at the second stage.

\subsection{IIP3 of Parallel Amplifiers}

The Type 3 VGA uses parallel amplifiers for the first and second stages, and thus the allotment of gain and linearity for the HGA and HLA has to be considered. To optimize the circuit parameters, we analyzed and calculated the gain and the IIP3 of the VGA.

The overall IIP3 of a cascaded amplifier, shown in Fig. 5(a), has been derived in Ref. [5].

$$
\frac{1}{i i p 3_{\text {Cas }}^{2}}=\frac{1}{i i p 3_{1}^{2}}+\frac{g_{1}^{2}}{i i p 3_{2}^{2}}
$$

$$
i i p 3_{\text {Cas }}=\sqrt{\frac{1}{\frac{1}{i i p 3_{1}^{2}}+\frac{g_{1}^{2}}{i i p 3_{2}^{2}}}},
$$

where iip $3_{C a s}, i i p 3_{1}$, and $i i p 3_{2}$ are the IIP 3 of the cascaded amplifier, the first stage, and the second stage in voltage domain, respectively, and $g_{1}$ and $g_{2}$ represent the voltage gains of the first and second stages, respectively.

On the other hand, there is no literature about the IIP3 of a parallel amplifier, as shown in Fig. 5(b). To derive the overall IIP3 of the parallel amplifier, consider two transconductors $\mathrm{gm}_{1}$ and $\mathrm{gm}_{2}$ connected in parallel and a common load resistor $R_{C}$, as shown in Fig. 5(c). In general, the IIP3 $[\mathrm{dBm}]$ is expressed as follows,

$$
I I P 3=\frac{P i+G-I M 3}{2}+P i,
$$

where Pi $[\mathrm{dBm}]$ and $\mathrm{G}[\mathrm{dB}]$ are the input power and power gain of the amplifier, respectively. Therefore, we have the third intermodulation IM3 [dBm]

$$
I M 3=3 P i+G-2 I I P 3 .
$$

Considering the first amplifier has power gain $G_{1}[\mathrm{~dB}]$ and IIP3 $3_{1}[\mathrm{dBm}]$, and the second one has $G_{2}[\mathrm{~dB}]$ and $\mathrm{IIP}_{2}[\mathrm{dBm}]$, we have

$$
I M 3_{1}=3 P i+G_{1}-2 I I P 3_{1}
$$

and

$$
I M 3_{2}=3 P i+G_{2}-2 I I P 3_{2} .
$$

The total $I M 3[\mathrm{dBm}]$ is obtained by the sum of both $I M 3_{1}$ and $I M 3_{2}$ in antilogarithms.

$$
\begin{aligned}
I M 3_{\text {tot }} & =10 \log \left(10^{\frac{3 P i+G_{1}-2 I I P 3_{1}}{10}}+10^{\frac{3 P i+G_{2}-2 I I P 3_{2}}{10}}\right) . \\
& =10 \log \left(\frac{g_{1}^{2}}{i i p 3_{1}^{4}}+\frac{g_{2}^{2}}{i i p 3_{2}^{4}}\right)+3 P i .
\end{aligned}
$$

The total power gain $G_{t o t}$ is

$$
\begin{aligned}
G_{t o t} & =20 \log \left\{R_{C}\left(g m_{1}+g m_{2}\right)\right\} \\
& =10 \log \left(g_{1}+g_{2}\right)^{2} .
\end{aligned}
$$

Note that $g_{1}, g_{2}, i i p 3_{1}$, and $i i p 3_{2}$ are voltage quantities rather than power quantities.

The overall IIP3 of the parallel amplifier IIP3 $3_{\text {para }}$ is obtained by substituting Eqs. (8) and (9) for Eq. (4).

$$
\begin{aligned}
I I P 3_{\text {para }}= & \frac{P i+10 \log \left(g_{1}+g_{2}\right)^{2}}{2} \\
& -\frac{10 \log \left(\frac{g_{1}^{2}}{i i p 3_{1}^{4}}+\frac{g_{2}^{2}}{i i p 3_{2}^{4}}\right)+3 P i}{2}+P i \\
= & 10 \log \frac{g_{1}+g_{2}}{\sqrt{\frac{g_{1}^{2}}{i i p 3_{1}^{4}}+\frac{g_{2}^{2}}{i i p 3_{2}^{4}}}}
\end{aligned}
$$


For the IIP3 in voltage domain, as shown in Eq. (3), we conclude that the overall IIP3 of the parallel amplifier is represented by

$$
i i p 3_{\text {para }}^{2}=\frac{g_{1}+g_{2}}{\sqrt{\frac{g_{1}^{2}}{i i p 3_{1}^{4}}+\frac{g_{2}^{2}}{i i p 3_{2}^{4}}}} .
$$
as

In general, the IIP3 of $\mathrm{n}$ parallel amplifiers is expressed

$$
i i p 3_{\text {para }}^{2}=\frac{\sum_{i=1}^{n} g_{i}}{\sqrt{\sum_{i=1}^{n} \frac{g_{i}^{2}}{i i p 3_{i}{ }^{4}}}} .
$$

\subsection{Analysis of VGA Gain and IIP3}

The gain and the IIP3 of a bipolar differential amplifier with and without degeneration resistors shown in Figs. 6(a) and 6(b) have been discussed in Refs. [6]-[8]. To calculate the

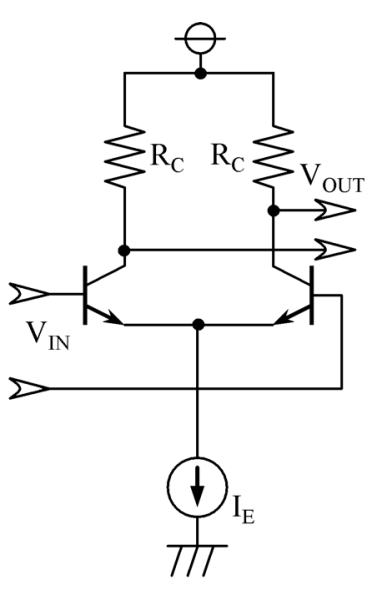

(a)

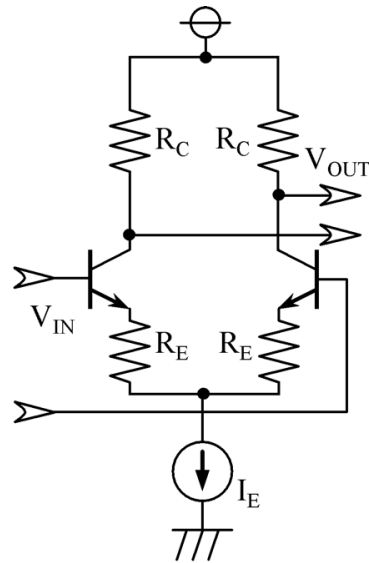

(b)

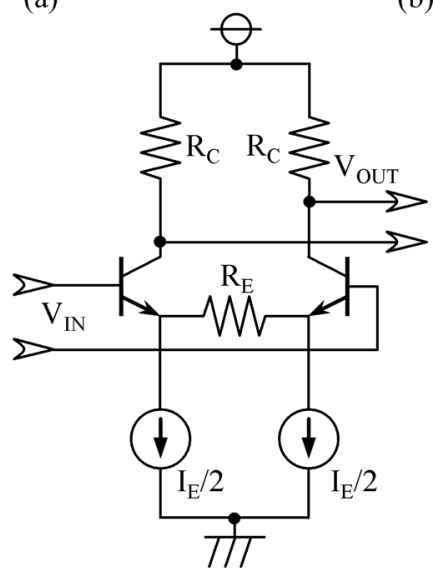

(c)

Fig. 6 (a) Simple differential amplifier, (b) emitter-degeneration differential amplifier, (c) simplified circuit of the parallel amplifier. gain and the IIP3 of the VGA in Fig. 3, we modified the equations in these works for the differential amplifier with a common emitter-degeneration resistor shown in Fig. 6(c), and used the equations discussed in Sect. 2.2.

For the simple differential amplifier without the degeneration resistor, the HGA, the voltage gain and the IIP3 are given by

$$
\begin{aligned}
& g_{H}=g_{m} R_{C}=\frac{I_{E} R_{C}}{2 V_{T}} \\
& i i p 3_{H}=4 V_{T},
\end{aligned}
$$

where $I_{E}, R_{C}$, and $V_{T}$ are the tail current, the load resistor, and the thermal voltage, respectively. Note that iip 3 is a zero-peak voltage. For the common emitter-degeneration differential amplifier shown in Fig. 6(c), the HLA, the gain and IIP3 are expressed as

$$
\begin{gathered}
g_{L}=g^{m} R_{C}=\frac{I_{E} R_{C}}{2 V_{T}+\frac{I_{E} R_{E}}{2}} \\
i i p 3_{L}=4 V_{T}\left(1+\frac{I_{E} R_{E}}{4 V_{T}}\right)^{\frac{3}{2}},
\end{gathered}
$$

where $R_{E}$ is the common emitter-degeneration resistor. The coefficients in Eqs. (15) and (16) are modified from the equations in Ref. [8].

Despite the fact that these formulas are available only for small input signals, they are effective in analyzing the VGA because the VGA operates in a linear region, and the IIP3 is defined at a small input signal.

The gain of the fully parallel-amplifier VGA (Type 3) is given by

$$
\begin{aligned}
g_{\text {type } 3}= & g_{H G A 1} g_{H G A 2}+g_{H L A 1} g_{H L A 2} \\
= & \frac{I_{1 H} R_{C 1 H}}{2 V_{T}} \frac{I_{2 H} R_{C 2}}{2 V_{T}} \frac{1}{1+\exp \left(-V_{c n t 2} / V_{T}\right)} \\
& +\frac{I_{1 L} R_{C 1 L}}{2 V_{T}+\frac{I_{L} R_{E 1}}{2}} \frac{I_{2 L} R_{C 2}}{2 V_{T}+\frac{I_{2 L} R_{E 2}}{2}} \frac{1}{1+\exp \left(V_{c n t 2} / V_{T}\right)},
\end{aligned}
$$

where $I_{1 H}, I_{1 L}, I_{2 H}$, and $I_{2 L}$ are the tail currents of the first and second stages, respectively, and $I_{2 H}$ and $I_{2 L}$ are constant currents. Here, $I_{1 H}$ and $I_{1 L}$ are variable currents expressed as

$$
\begin{aligned}
& I_{1 H}=\frac{I_{1 H 0}}{1+\exp \left(-V_{c n t 1} / V_{T}\right)} \\
& I_{1 L}=\frac{I_{1 L 0}}{1+\exp \left(V_{c n t 1} / V_{T}\right)} .
\end{aligned}
$$

The control voltages $V_{c n t 1}$ and $V_{c n t 2}$ are converted from control voltage $V_{\text {cont }}$ using a control voltage generator, which will be discussed in the following section.

The IIP3 of the Type 3 VGA can be obtained as follows. First, derive the IIP3 of the high gain path (HGP) which consists of HGA1 and HGA2, and of the high linear path (HLP) which is composed of HLA1 and HLA2. Then, calculate the total IIP3 using Eq. (11) because the Type 3 VGA is regarded as a parallel amplifier to HGP and HLP. The IIP3s of 


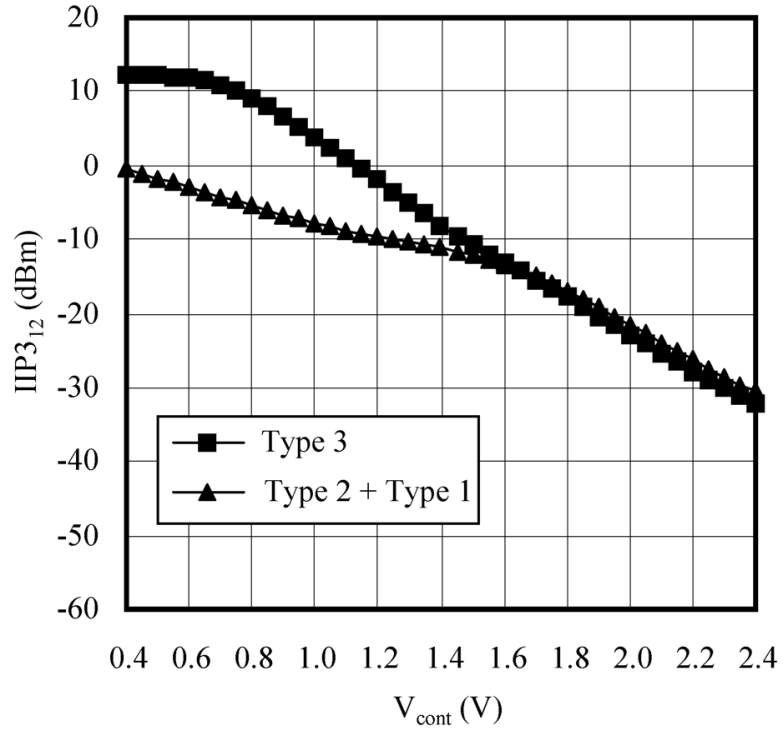

Fig. 7 IIP3 of the 1st-2nd stage versus Vcont.

HGP and HLP are given by

$$
\begin{aligned}
& i i p 3_{H G P}=\sqrt{\frac{1}{\frac{1}{i i p 3_{H G A 1}^{2}}+\frac{g_{H G A 1}^{2}}{i i p 3_{H G A 2}^{2}}}} \\
& =\frac{4 V_{T}}{\sqrt{1+\left(\frac{I_{1 H} R_{C 1 H}}{2 V_{T}}\right)^{2}}} \\
& i i p 3_{H L P}=\sqrt{\frac{1}{\frac{1}{i i p 3_{H L A 1}^{2}}+\frac{g_{H L A 1}^{2}}{i i p 3_{H L A 2}^{2}}}}
\end{aligned}
$$

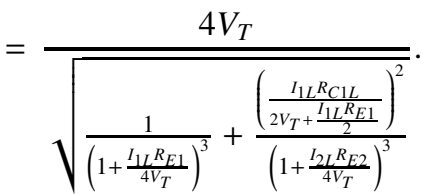

Finally, the overall IIP3 of the Type 3 VGA is obtained by substituting Eqs. (20) and (21) for Eq. (11).

$$
i i p 3_{\text {type }}^{2}=\frac{g_{H G A 1} g_{H G A 2}+g_{H L A 1} g_{H L A 2}}{\sqrt{\frac{\left(g_{H G A 1} g_{H G A 2}\right)^{2}}{i i p 3_{H G P}^{4}}+\frac{\left(g_{H L A 1} g_{H L A 2}\right)^{2}}{i i p 3_{H L P}^{4}}}}
$$

In the same way, we can calculate the total gain and the IIP3 of the four-stage VGA.

Figure 7 shows an example of the IIP3 comparison between the Type 3 VGA and a conventional VGA (Type $2+$ Type 1) under the conditions that the gain variation range is $40 \mathrm{~dB}$, and $i i p 3_{H G A 1}$, and $i i p 3_{H L A 1}$ are $0.104 V_{0-p}$ and $2.05 V_{0-p}$, respectively. In spite of having the same IIP3 as the first stage of the Type 3 VGA, the total IIP3 of the conventional VGA is much lower than that of the Type $3 \mathrm{VGA}$ at the low control voltage region. This is because the third-order distortion current generated by the HGA becomes dominant, as described in Sect. 2.1. Figure 8 shows a comparison of the total IIP3 for the 4-stage VGA. The Type

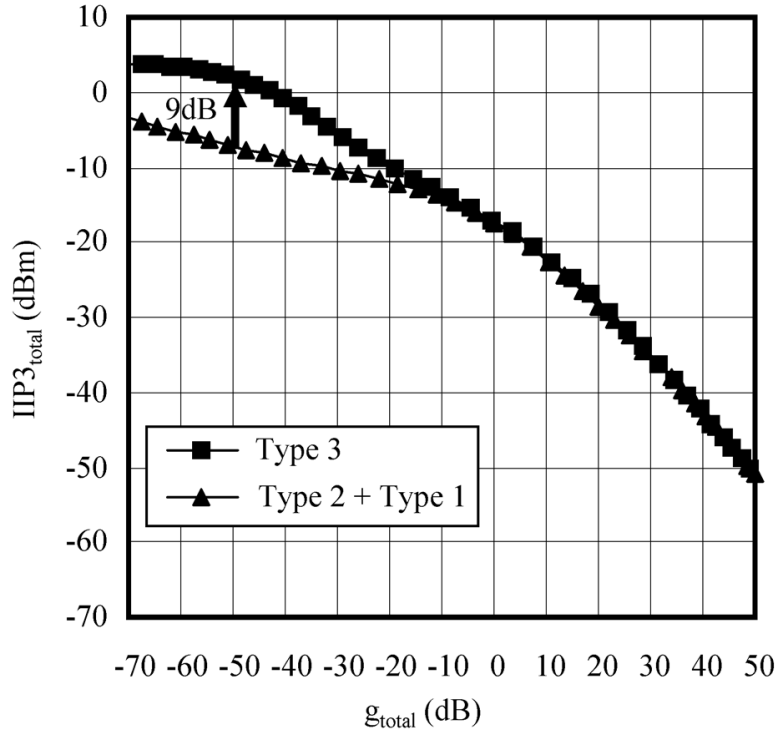

Fig. 8 Total IIP3 versus total gain of 4-stage VGA.

1 VGAs are used for the third and fourth stages. The total IIP3 of the Type 3 VGA is improved by $9 \mathrm{~dB}$ at the total gain of $-50 \mathrm{~dB}$. In the high gain region over $-10 \mathrm{~dB}$, both IIP3s show the same characteristics because the non-linearity of the high gain amplifiers becomes dominant. In this region, the degradation of the IIP3 does not have a harmful influence on the circuit because the input signal swing is much smaller than the IIP3.

\subsection{Temperature Compensation}

Since the transmission and the reception are done alternately in the Time Division Duplex (TDD), the calibration can be performed before the period of the switch of the transmission and the reception. On the other hand the communication is continuous in the Frequency Division Duplex (FDD). Therefore, a continuous temperature compensation is required to correspond to the temperature change during the communication. Figure 9 shows a control voltage generator to compensate for the temperature dependence of the VGA. First, with the V-I converter which is composed of a voltage follower and a resistor $R_{V I}$, the gain control voltage $V_{\text {cont }}$ is converted to the current $I_{V I}$ proportional to $V_{\text {cont }}$. The output current $I_{V I}$ is expressed as

$$
I_{V I}=k_{V I} \frac{V_{\text {cont }}}{R_{V I}}
$$

where $k_{V I}$ is a constant coefficient. Second, the current converter outputs the currents $I_{c n t}$ and $I_{c n t b}$ in proportion to both T and $V_{\text {cont }}$. The $I_{c n t}$ and $I_{c n t b}$ are connected to HLA2, HGA2, HGA3, and HGA4 through current mirror circuits, and make the control voltage $V_{c n t}$ and $V_{c n t 2}$ in Figs. 1 and 3 with resistors $R_{c n t}$ and $R_{c n t 2}$, which are not shown in the figure. Finally, the current is converted as proportional to $\mathrm{T}$ times the exponential of $T V_{\text {cont }}$ over $V_{T}$ by the exponential converter. The output current $I_{\text {ecnt }}$ and $I_{\text {ecntb }}$ supply the 


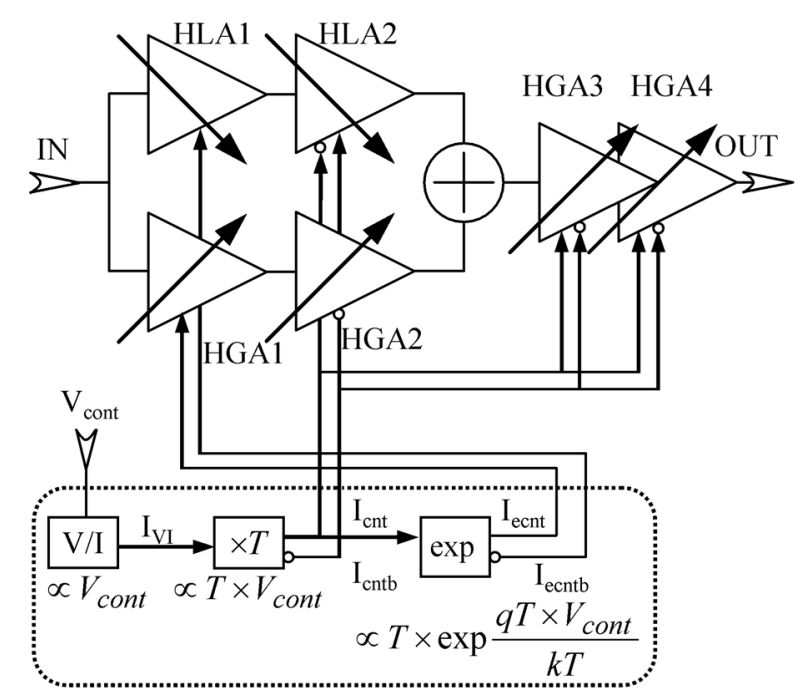

Fig. 9 Control voltage generator.

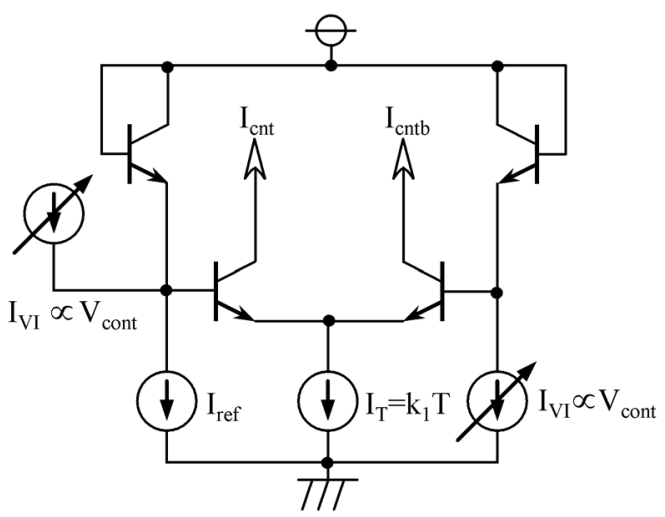

Fig. 10 Current converter to temperature proportional current.

tail current for HGA1 and HLA1 through current mirror circuits, respectively.

Figure 10 depicts a simplified circuit of the current converter based on a current multiplier [9]. The $I_{c n t}$ and $I_{c n t b}$ are the output current, $I_{V I}$ is the output current of the V-I converter, and $I_{r e f}$ is the constant current. The $I_{T}$ is the tail current for the differential pair, and is in proportion to T. Both $I_{r e f}$ and $I_{T}$ are made by a bandgap reference circuit, current mirrors, and resistors. The relations between each current are expressed in the following equations.

$$
\begin{aligned}
& I_{c n t}: I_{c n t b}=I_{V I}:\left(I_{r e f}-I_{V I}\right) \\
& I_{c n t b}=I_{T}-I_{c n t} .
\end{aligned}
$$

From Eqs. (24) and (25), one of the output current $I_{c n t}$ is given by

$$
I_{c n t}=\frac{I_{T}}{I_{\text {ref }}} I_{V I}=\frac{k_{1} T}{I_{r e f}} \frac{k_{V I} V_{c o n t}}{R_{V I}},
$$

where $I_{r e f}$ is constant, and $I_{T}$ and $I_{V I}$ are proportional to T and $V_{\text {cont }}$, respectively, and $k_{1}$ is a constant coefficient. Thus, $I_{c n t}$ is proportional to both $\mathrm{T}$ and $V_{\text {cont }}$.

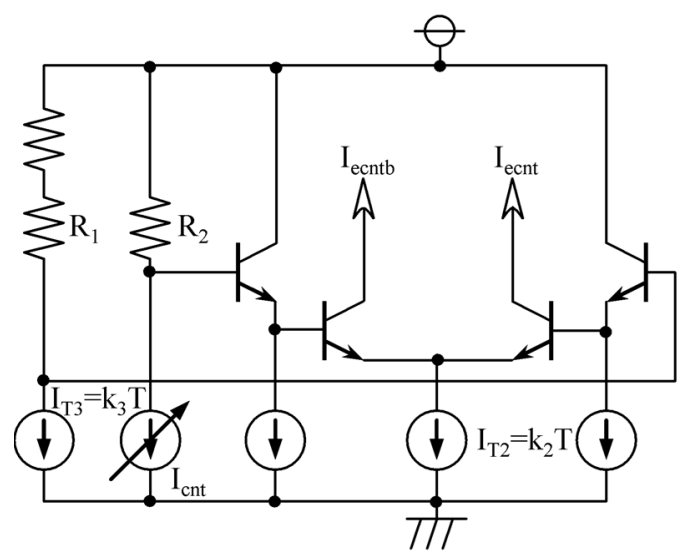

Fig. 11 Exponential converter.

The control voltage $V_{c n t}$ and $V_{c n t 2}$ are given by

$$
\begin{aligned}
V_{c n t(c n t 2)} & =R_{\text {cnt(cnt2) }}\left(I_{c n t}-I_{c n t b}\right) \\
& =R_{\text {cnt }(c n t 2)}\left(2 I_{c n t}-I_{T}\right) \\
& =R_{c n t(c n t 2)} k_{1} T\left(\frac{2}{I_{r e f}} \frac{k_{V I} V_{c o n t}}{R_{V I}}-1\right)
\end{aligned}
$$

where $R_{c n t}$ and $R_{c n t 2}$ are the resistors which define the control voltage. From Eq. (1), the current transfer is expressed as

$$
\begin{aligned}
\frac{I_{c 1}}{I_{e}} & \cong \exp \left(\frac{R_{c n t}(c n t 2) k_{1} T\left(\frac{2}{I_{\text {ref }}} \frac{k_{V I} V_{\text {cont }}}{R_{V I}}-1\right)}{V_{T}}\right) \\
& =\frac{\exp \left(R_{\text {cnt }(c n t 2)} k_{1} \frac{q}{k} \frac{2}{I_{\text {ref }}} \frac{k_{V I} V_{c o n t}}{R_{V I}}\right)}{\exp \left(R_{\text {cnt }(c n t 2)} k_{1} \frac{q}{k}\right)} .
\end{aligned}
$$

Equation (28) shows that the current transfer, or the gain of the second, third, and fourth, is proportional to $\exp \left(V_{\text {cont }}\right)$ and has no temperature dependence.

Figure 11 shows a simplified circuit of the exponential converter, which consists of a differential pair with the tail current $I_{T 2}$ proportional to T. One of the output current $I_{e c n t}$ is given by

$$
\begin{aligned}
I_{\text {ecnt }} & \cong I_{T 2} \exp \left(\frac{I_{c n t} R_{2}-I_{T 3} R_{1}}{V_{T}}\right) \\
& =k_{2} T \exp \left(\frac{k_{V I} k_{1} R_{2} q}{I_{r e f} R_{V I} k} V_{c o n t}-\frac{k_{3} R_{1} q}{k}\right)
\end{aligned}
$$

where $I_{T 3}$ is bias current proportional to $\mathrm{T}$, and $k_{2}$ and $k_{3}$ are constant coefficients for $I_{T 2}$ and $I_{T 3}$, respectively. Equation (29) indicates that $I_{e c n t}$ is proportional to T times the exponential of $V_{\text {cont }}$. The current $I_{\text {ecntb }}$ is the difference between $I_{T 2}$ and $I_{\text {ecnt }}$.

The output current $I_{\text {ecnt }}$ and $I_{\text {ecntb }}$ are mirrored to the tail current of the first stage amplifiers $I_{1 H}$ and $I_{1 L}$ in Fig. 3, respectively. Using Eqs. (13) and (15), the gain of HGA1 and HLA1 is given by

$$
g_{H G A 1}=\frac{q I_{1 H} R_{C 1 H}}{2 k T}=a \frac{q k_{2} R_{C 1 H}}{2 k} \exp \frac{b q V_{c o n t}}{k},
$$




$$
g_{H L A 1}=\frac{I_{1 L} R_{C 1 L}}{\frac{2 k T}{q}+\frac{I_{1 L} R_{E 1}}{2}}=\frac{R_{C 1 L}}{\frac{2 k}{c q k_{2} \exp \left(-\frac{b q V_{c o n t}}{k}\right)}+\frac{R_{E 1}}{2}}
$$

where $\mathrm{a}, \mathrm{b}$, and $\mathrm{c}$ are constant coefficients which are design parameters. Since both $g_{H G A 1}$ and $g_{H L A 1}$ do not include the term $\mathrm{T}$, a temperature independent gain is obtained.

In fact, each bias current, $I_{r e f}, I_{T}, I_{T 2}$, and $I_{T 3}$, is determined by a resistor. Therefore, $I_{r e f}, k_{1}, k_{2}$ and $k_{3}$ are proportional to the reciprocal of the resistor value which has temperature dependence. However, $R_{\text {cnt(cnt2) }} k_{1}$ and $I_{\text {ref }} R_{V I}$ in Eq. (28), $k_{2} R_{C 1 H}$ in Eq. (30), and $k_{2} R_{C 1 L}$ in Eq. (31) become constant because the temperature coefficient of each resistor is canceled.

\section{Experimental Results and Comparisons}

The VGA was fabricated using a $0.5 \mu \mathrm{m}$ Bi-CMOS process that has $26 \mathrm{GHz}_{\mathrm{T}}$ npn transistors, lateral pnp transistors, and CMOS transistors. A die photograph is shown in Fig. 12. The chip consists of a pair of 4-stage VGAs and a demodulator, PLLs, and a transmitter, which comprise an IFIC for WCDMA [3]. The chip occupies $4 \times 4 \mathrm{~mm}^{2}$. To improve the noise and linearity performance, circuit parameters, such as degeneration resistors, decoupling capacitors for bias circuits and resistors of the control voltage generator, were slightly changed from those of Ref. [3].

Figure 13 shows the relation between the measured total voltage gain and the control voltage $V_{\text {cont }}$ at $190 \mathrm{MHz}$ input. The total voltage gain includes the VGA gain, the demodulator gain of $40 \mathrm{~dB}$, and the $8 \mathrm{~dB}$ matching gain of an external matching circuit for measurement. A variable range of more than $110 \mathrm{~dB}(-28$ to $83 \mathrm{~dB})$ was obtained. The gain variation for temperature is shown in Fig. 14. The gain variation from $-30^{\circ}$ to $85^{\circ} \mathrm{C}$ was found to be within $1.7 \mathrm{~dB}$, which means that temperature variation was less than $0.014 \mathrm{~dB} /{ }^{\circ} \mathrm{C}$ over the entire dynamic range.

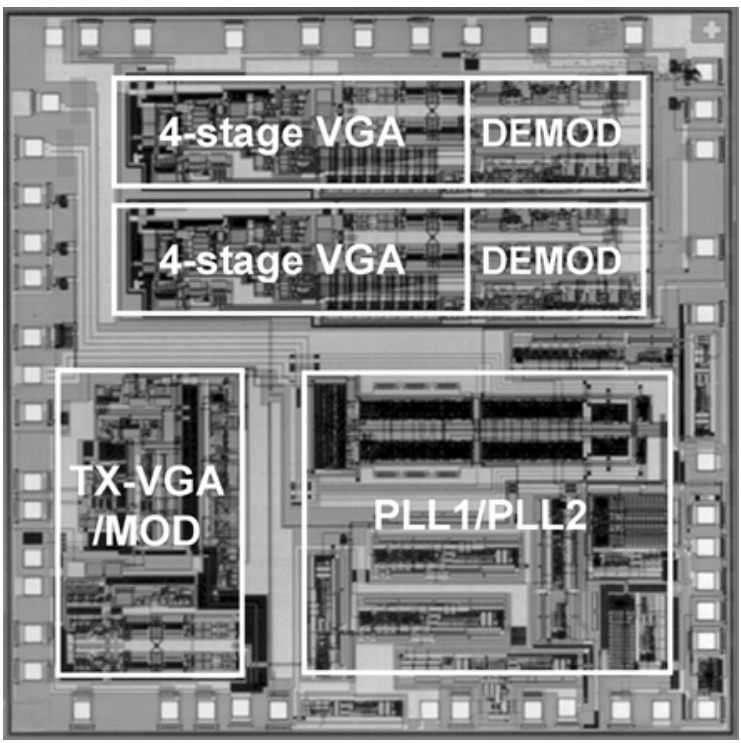

Fig. 12 Die photograph of the VGA.
The noise figure versus the voltage gain is shown in Fig. 15. The NF at $80 \mathrm{~dB}$ gain is $5.1 \mathrm{~dB}(5.3 \mathrm{~dB}$ in Ref. [3] at room temperature. This varies $\pm 0.2 \mathrm{~dB}$ at $-30^{\circ} \mathrm{C}$ and $85^{\circ} \mathrm{C}$.

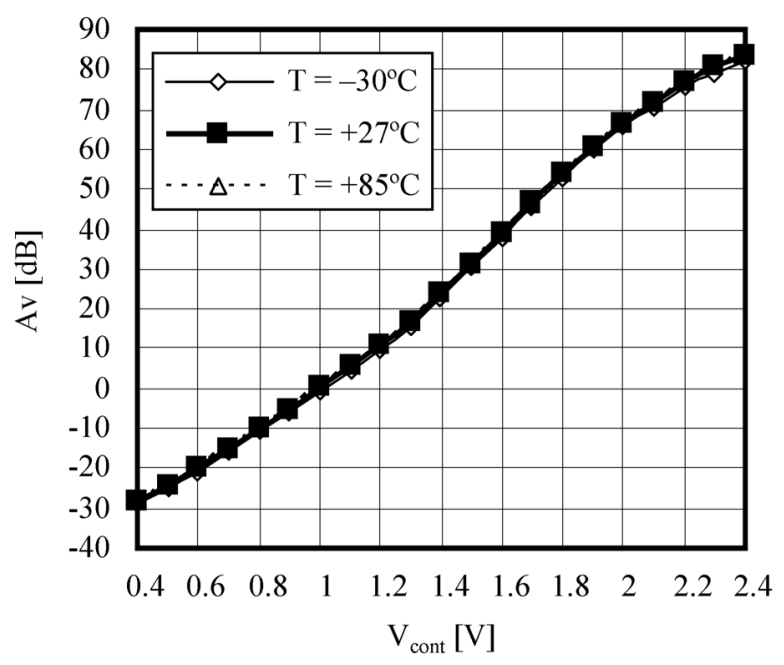

Fig. 13 Measured voltage gain versus control voltage ( $\mathrm{f}=190 \mathrm{MHz}, \mathrm{Vcc}$ $=3.0 \mathrm{~V})$.

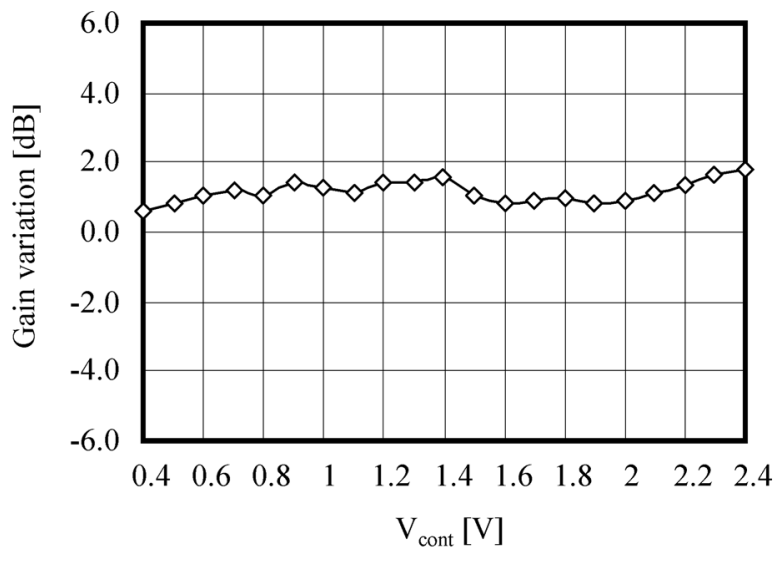

Fig. 14 Gain variation from $-30^{\circ} \mathrm{C}$ to $+80^{\circ} \mathrm{C}$.

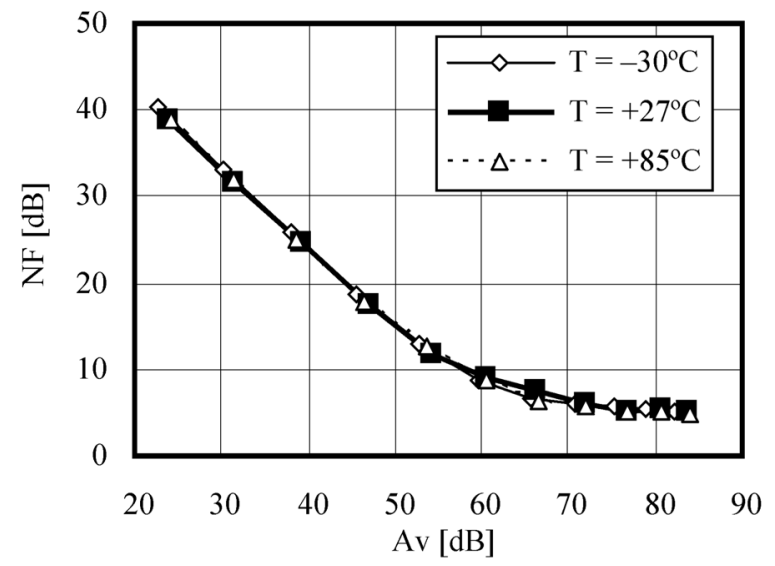

Fig. 15 Measured NF versus voltage gain $(f=190 \mathrm{MHz}, \mathrm{Vcc}=3.0 \mathrm{~V})$. 
Table 1 Performance summary and comparison of VGAs.

\begin{tabular}{|l|c|c|c|c|c|c|}
\hline & This work & $2000[10]$ & $2003[11]$ & $2003[12]$ & $2002[13]$ & $2007[14]$ \\
\hline Technology & $0.5 \mu \mathrm{m}$ BiCMOS & BiCMOS & $0.5 \mu \mathrm{m}$ BiCMOS & $0.18 \mu \mathrm{m}$ CMOS & $0.25 \mu \mathrm{m}$ CMOS & $0.18 \mu \mathrm{m}$ CMOS \\
Frequency [MHz] & $<600$ & $<500$ & 50 & 380 & 380 & $<900$ \\
Gain range [dB] & 111 & 78 & 98 & 73 & 80 & 94 \\
NF @Gmax [dB] & 5.1 & 5 & 4.9 & 5 & 11 & 6.8 \\
IP1dB [dBm] & -11 & $($ IIP3 - -8) & -15 & -18.6 & - & -11 \\
Current [mA] & $4.4-6.6$ & 12 & 13 & 6 & 25.3 & 11.4 \\
\hline
\end{tabular}

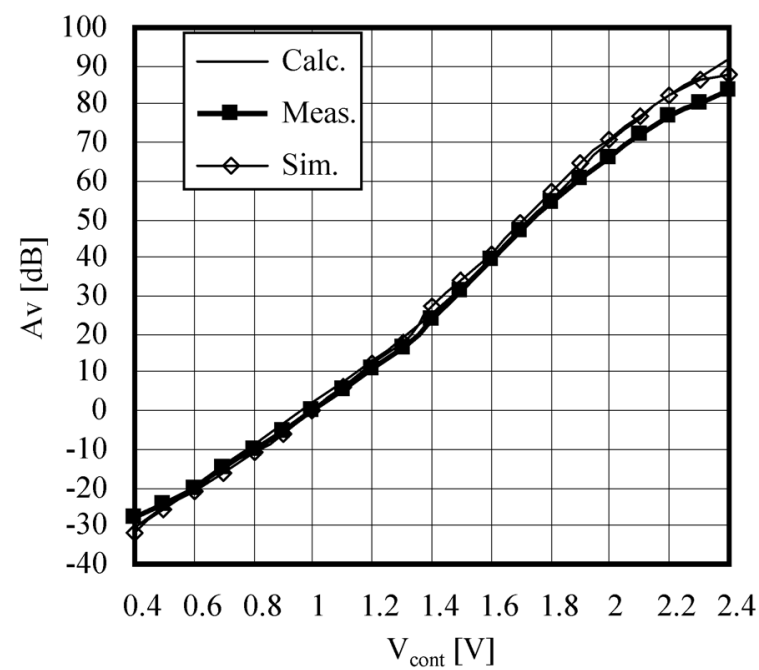

Fig. 16 Voltage gain versus control voltage $(\mathrm{f}=190 \mathrm{MHz}, \mathrm{Vcc}=3.0 \mathrm{~V}$, $\left.\mathrm{T}=27^{\circ} \mathrm{C}\right)$.

To confirm the circuit analysis, calculations using the equations were compared with the measurement and circuit simulation results. Figure 16 shows the total voltage gain versus $V_{\text {cont }}$ of calculation, measurement, and simulation. The demodulator gain of $40 \mathrm{~dB}$ and the matching gain of $8 \mathrm{~dB}$ are simply added to the calculation. Close agreement was found between the calculated, measured, and simulated results. Although the circuit analysis is simply a small signal DC analysis, it is effective in estimating the characteristics of the VGAs at an operating frequency below the $3 \mathrm{~dB}$ bandwidth of $600 \mathrm{MHz}$.

The same comparison for input $1 \mathrm{~dB}$ compression point IP1 dB versus voltage gain is shown in Fig. 17. The calculated IP1 dB is obtained by simply subtracting the matching gain of $8 \mathrm{~dB}$ and $9.6 \mathrm{~dB}$ from the calculated IIP3 [5]. The calculated result shows a good fit with the simulated and measured results. The difference between the calculated results and others at the high gain region of more than $50 \mathrm{~dB}$ is due to the non-linearity of the emitter followers of the fourth stage, which is not taken into account in the calculation. As mentioned in Sect. 2.3, the degradation of IP1 dB in this region does not have a harmful influence on the circuit. The circuit parameters, such as degeneration resistors and bias resistors of the control voltage generator, were redesigned

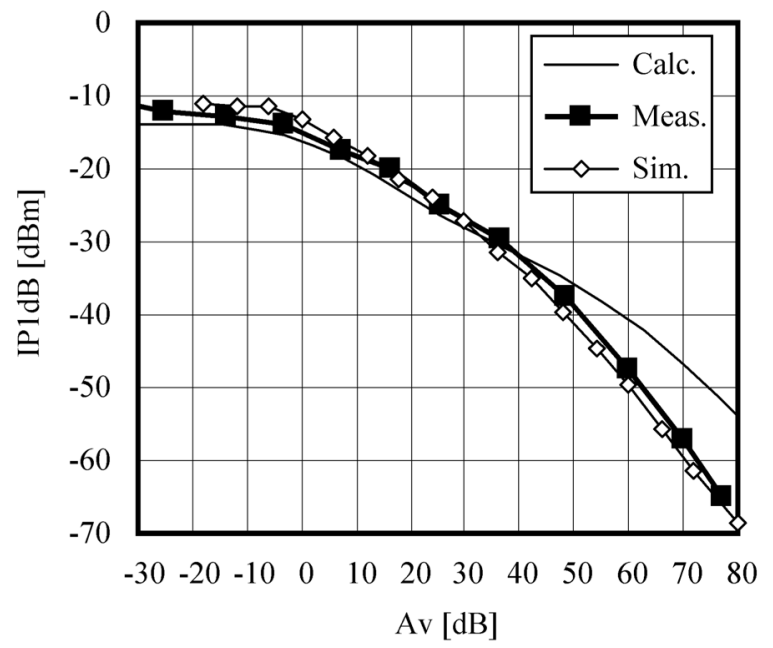

Fig. 17 Input $\mathrm{P} 1 \mathrm{~dB}$ variation versus voltage gain ( $\mathrm{f}=190 \mathrm{MHz}, \mathrm{Vcc}=$ $3.0 \mathrm{~V}, \mathrm{~T}=27^{\circ} \mathrm{C}$ ).

using the linearity analysis and circuit simulation. The measured maximum input $\mathrm{P} 1 \mathrm{~dB}$ was $-11 \mathrm{dBm}(-15 \mathrm{dBm}$ in Ref. [3]) at minimum gain.

With a $3 \mathrm{~V}$ power supply, the current consumption of the VGA varies from $4.4 \mathrm{~mA}$ (minimum gain) to $6.6 \mathrm{~mA}$ (maximum gain) including the control voltage generator and bias circuits. Table 1 summarizes the measurement results of the VGA and comparison with previous studies.

\section{Conclusions}

A wide-dynamic-range four-stage VGA with temperature compensation and an analysis of the gain and linearity of the VGA have been presented. The VGA using parallel amplifiers for the first and second amplifiers achieves a low NF in the high gain region and a high linearity in the low gain region. The input $\mathrm{P} 1 \mathrm{~dB}$ at minimum gain is improved by $9 \mathrm{~dB}$ compared with a conventional VGA. The VGA has a $111 \mathrm{~dB}$ gain control range with a maximum current of $6.6 \mathrm{~mA}$. In addition, the analysis of gain and linearity using the newly derived IIP3 for parallel amplifiers shows close agreement with measured and simulated results. 


\section{References}

[1] G.K.S. Sahota and C.J. Persico, "High dynamic range variable-gain amplifier for CDMA wireless applications," ISSCC Dig. Tech. Papers, pp.374-375, Feb. 1997.

[2] K. Lee, J. Park, J. Lee, S. Lee, H. Huh, D. Jeong, and W. Kim, "A single-chip $2.4 \mathrm{GHz}$ direct-conversion CMOS receiver for wireless local loop using one-third frequency local oscillator," Symp. VLSI circuits Dig. of Tech., pp.42-45, June 2000.

[3] T. Maruyama, K. Kaneki, K. Takahashi, H. Sato, T. Iga, and N. Kato, "Single-chip IF transceiver IC with wide dynamic range variable gain amplifiers for wideband CDMA applications," Symp. VLSI circuits Dig. of Tech., pp.11-14, June 2001.

[4] W.R. Davis and J.E. Solomon, "A high-performance monolithic IF amplifier incorporating electric gain control," IEEE J. Solid-State Circuits, vol.SC-3, no.4, pp.408-416, Dec. 1968.

[5] B. Razavi, RF Microelectronics, Prentice-Hall, Englewood Cliffs, NJ, 1997.

[6] W.M.C. Sansen and R.G. Meyer, "Distortion in bipolar transistor variable-gain amplifiers," IEEE J. Solid-State Circuits, vol.SC-8, no.4, pp.275-282, Aug. 1973.

[7] P.R. Gray, P.J. Hurst, S.H. Lewis, and R.G. Meyer, Analysis and Design of Analog Integrated Circuits, 4th ed., Wiley, New York, 2001.

[8] A.A. Abidi, "General relations between IP2, IP3, and offsets in differential circuits and the effects of feedback," IEEE Trans. Microw. Theory Tech., vol.51, no.5, pp.1610-1612, May 2003.

[9] B. Gilbert, “A new wide-band amplifier technique," IEEE J. SolidState Circuits, vol.SC-3, no.4, pp.353-365, Dec. 1968.

[10] S. Otaka, G. Takemura, and H. Tanimoto, "A low-power low-noise accurate linear-in-dB variable-gain amplifier with $500-\mathrm{MHz}$ bandwidth,” IEEE J. Solid-State Circuits, vol.35, no.12, pp.1942-1948, Dec. 2000.

[11] K. Nah and B. Park, "A 50-MHz dB-linear programmable-gain amplifier with 98-dB dynamic range and 2-dB gain steps for $3 \mathrm{~V}$ power supply," IEEE Trans. Very Large Scale Integr. (VLSI) Syst., vol.11, no.2, pp.218-223, April 2003.

[12] R. Saito, K. Hosoda, A. Hyogo, T. Maruyama, H. Komurasaki, H. Sato, and K. Sekine, "A 1.8-V 73-dB dynamic-range CMOS variable gain amplifier,' Proc. 29th ESSCIRC, pp.301-304, Sept. 2003.

[13] O. Watanabe, S. Otaka, M. Ashida, and T. Itakura, "A 380-MHz CMOS linear-in-dB signal-summing variable gain amplifier with gain compensation techniques For CDMA systems," Symp. VLSI Circuits Dig. Tech., pp.136-139, June 2002.

[14] H. Lee, K. Lee, and S. Hong, "A wideband CMOS variable gain amplifier with an exponential gain control," IEEE Trans. Microw. Theory Tech., vol.55, no.6, pp.1363-1373, June 2007.

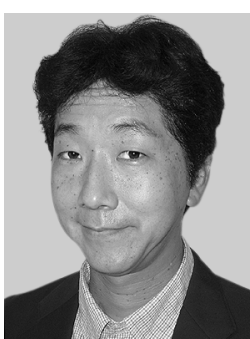

Hisayasu Sato received the B.S. degree in physics from Osaka University, Osaka, Japan, in 1982. In 1982, he joined the LSI Research and Development Laboratory, Mitsubishi Electric Corporation, Itami, Japan, where he was engaged in development of high-speed communication LSI's and CMOS/BiCMOS RF transceivers. Since 2010, he has been working for Renesas Electronics Corporation, Itami, Japan. Currently he is managing the research and development of high frequency analog integrated circuit design. Mr. Sato is a member of the IEEJ, and the IEEE.

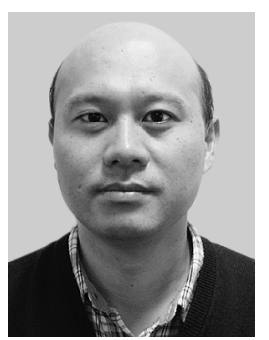

Takaya Maruyama was born in Wakayama, Japan, in 1970. He received the B.S. degree and M.S. degree in Engineering degree in Electrical Engineering from Osaka Prefecture University, Osaka, Japan in 1993 and 1995, respectively. He joined Mitsubishi Electric Corp. in 1995, where he developed BiCMOS transceivers. He transferred to Renesas Electronics Corp. in 2010. His research interest is in the area of CMOS RF transceiver circuit design.

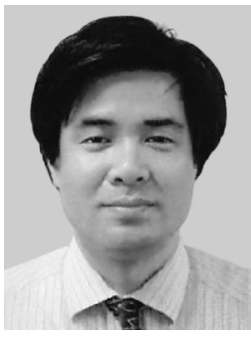

Toshimasa Matsuoka received the B.S. M.S. and Ph.D. degrees in electronic engineering from Osaka University, Osaka, Japan, in 1989, 1991 and 1996, respectively. During 1991-1998, he worked for the Central Research Laboratories, Sharp Corporation, Nara, Japan, where he was engaged in the research and development of deep submicron CMOS devices and ultra thin gate oxides. Since 1999, he has been working for Osaka University, where he is Associate Professor now. His current research includes CMOS RF circuits and device modeling. Dr. Matsuoka is a member of the Japan Society of Applied Physics, the IEEE, and the IEEJ.

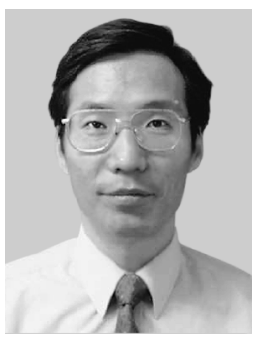

Kenji Taniguchi received the B.S., M.S. and Ph.D. degrees from Osaka University, Osaka, Japan, in 1971, 1973 and 1986 respectively. From 1973 to 1986, he worked for Toshiba Research and Development Center, Kawasaki, Japan, where he was engaged in process modeling and the design of MOS LSI fabrication technology. He was a Visiting Scientist at Massachusetts Institute of Technology, Cambridge, from July 1982 to November 1983. Presently, he is a Professor of Electronics Engineering at Osaka University. His current research interests are in analog circuits, radio frequency circuits, device physics and process technology. Prof. Taniguchi is a member of the Japan Society of Applied Physics and the IEEJ. He is a fellow of the IEEE. 